$$
\begin{gathered}
s^{2} \sigma^{2}+2 \rho b+a^{2}+\left(s^{2}+\sigma^{2}\right) \rho^{2}-s \sigma(s+\sigma) 2 \rho \\
+s \sigma\left(2 a-2 \rho^{2}\right)+(s+\rho)(2 \rho a+2 b)=0 \\
v^{2} s^{2}+2 a v s+2 b(v+s)+a^{2}=0 \\
w^{2} \sigma^{2}+2 a w \sigma+2 b(w+\sigma)+a^{2}=0
\end{gathered}
$$

Elimination of $s$ from (15) and (17) gives an equation symmetrical in $v$ and $\sigma$, which is thus identical with the result of eliminating $w$ from (27) and (18), so that (27) is consistent with (15), (17) and (18). It may be shown by actual division that the square of (27) is a factor of (23). In carrying out the division, the symmetry of (27) as to $\rho, v$ and $w$, in connection with the assumed symmetry of (23) in the same quantities, permits many terms of the quotient to be written by inspection after a few have been obtained. At this stage the knowledge of the factors for the special case $\rho=0$ determines many coefficients in the general case. The entire computation has been carefully checked, and the single assumption of symmetry verified, but the length of the quotient (one hundred ninety-eight terms) prevents its reproduction in this paper. It is of particular importance that this identical transformation, when employed to obtain new curves from the $\wp$-function curves in connection with the theory of conjugate functions, reproduces the original curves.

University of Pennsyltania, April, 1911.

\title{
SURFACES IN HYPERSPACE WHICH HAVE A TAN- GENT LINE WITH THREE-POINT CONTACT PASSING THROUGH EACH POINT.
}

\author{
BY PROFESSOR C. L. E. MOORE.
}

(Read before the American Mathematical Society, December 27, 1911.)

THROUGH each point on a surface in ordinary space $S_{3}$ pass two tangents having with the surface three-point contact (tangents to the asymptotic lines). The osculating planes to these curves are also tangent to the surface at the point of osculation. It is easily seen that the lines on a ruled surface in hyperspace have these same properties. The question 
then naturally arises whether there exist in hyperspace surfaces not ruled having the same sort of lines. It is the object of this paper to show the existence of such surfaces and to derive the differential equation which the coordinates must satisfy. The results are generalized for spreads of higher dimensions than two.

1. The existence of such surfaces can be shown geometrically as follows: On a ruled surface in a space $S_{n}$ trace three infinitely near curves $c, c_{1}, c_{2}$. Through the curves $c_{1}$ and $c_{2}$ pass a second ruled surface infinitely near the first and on this surface trace a third curve $c_{3}$ infinitely near to $c_{1}, c_{2}$. Then through the curves $c_{2}, c_{3}$ pass a third ruled surface infinitely near to the second and on it trace a curve $c_{4}$ infinitely near $c_{2}, c_{3}$. Continuing this process to the limit, it is at once evident that the locus of the curves $c$ is a surface of the kind desired.

We will now derive the condition that a surface in $S_{n}$ shall have through each point a curve whose osculating plane is tangent to the surface.

Let the surface be

$$
x^{(i)}=x^{(i)}(u, v) \quad(i=1,2,3, \cdots, n+1)
$$

and let three neighboring points be

$$
\text { (2) } x, \quad \text { (3) } x_{1} d u+x_{2} d v \text {,* }
$$

$$
x_{11} d u^{2}+2 x_{12} d u d v+x_{22} d v^{2}+x_{1} d^{2} u+x_{2} d^{2} v .
$$

The tangent plane to the surface at the point $x$ is defined by the three points†

$$
x, x_{1}, x_{2} \text {. }
$$

If the given curve is to have this plane for osculating plane, it is only necessary that the point

$$
x_{11} d u^{2}+2 x_{12} d u d v+x_{22} d v^{2}
$$

should lie in it; for the points (2), (3), and $x_{1} d^{2} u+x_{2} d^{2} v$ already lie in it. The condition then that (4) should lie in the tangent plane is

$$
\text { (6) } x_{11} d u^{2}+2 x_{12} d u d v+x_{22} d v^{2}+A x_{1}+B x_{2}+C x=0 \text {. }
$$

* Subscripts denote derivatives with respect to a variable $t$, where the curve on the surface is defined by assuming $u$ and $v$ to be functions of $t$. Hereafter superscripts will be omitted where no ambiguity occurs.

$\dagger$ See Segre, "Su una classa di superficie degl'iperspazii ecc.", Atti di Torino, 1907. 
If the coordinates satisfy (6), then the points (2), (3), (4) will lie in the tangent plane. Hence:

If the coordinates of a surface in $S_{n}$ satisfy a parabolic* partial differential equation of the second order, then through each point of the surface passes a curve whose osculating plane coincides with the tangent plane to the surface at the point, and conversely.

If now a tangent line is to have three-point contact with the surface, two conditions are necessary: $(a)$ the point (5) must lie in the tangent plane; (b) for some choice of $d^{2} u: d^{2} v$ the point (4) must lie on the tangent line. The point (4) lies on the line joining (5) to $x_{1} d^{2} u+x_{2} d^{2} v$, and consequently if (6) is satisfied then when $d^{2} u: d^{2} v$ varies the point (4) will describe a line in the tangent plane. This line will cut the tangent line drawn in the direction $d u / d v$. Thus if condition $(a)$ is satisfied, condition $(b)$ is also satisfied. Hence:

The necessary and sufficient condition that a surface in $S_{n}$ have through each point a tangent line having three-point contact with the surface is that the coordinates of the surface satisfy a parabolic partial differential equation of the second order.

From the form of equation (6) it is seen that the directions of the three-point tangents are in the direction of the characteristics, since each is in the direction defined by $d u / d v$.

In the paper referred to above Segre showed that a surface whose coordinates are particular solutions of two general partial differential equations of the second order must either be a developable or else lie in an ordinary space of three dimensions. From the above property it is evident that the surface could not be a developable if the coordinates satisfy two parabolic differential equations, for that would necessitate two principal directions (directions of three-point contact), which would be impossible. Then if such be the case, the surface must lie in an ordinary space $S_{3}$. In the pencil of partial differential equations which is defined by two general ones there are always two parabolic ones. Hence the two partial differential equations which the coordinates of a developable surface in hyperspace satisfy cannot be independent but must be such that there is only one parabolic equation belonging to the pencil defined by them.

2. The above considerations can be extended to varieties

* See Segre, loc. cit., $\$ 15$. 
of any number of dimensions contained in a space $S_{n}$ of $n$ dimensions. Let the coordinates of the variety $V_{m}$ be

$$
x^{(i)}=x^{(i)}\left(u_{1} u_{2} \cdots u_{m}\right) \quad(i=1,2, \cdots, n+1) .
$$

The tangent $S_{m}$ to $V_{m}$ is defined by the points

$$
x, \quad x_{1}, \quad x_{2}, \cdots, \quad x_{m}
$$

and if the osculating plane to a curve traced on the variety lies in this tangent $S_{m}$, then the three points

$$
\begin{aligned}
& \text { (8) } x, \quad \text { (9) } \sum_{i=1}^{i=m} x_{i} d u_{i}, \\
& \sum_{i=1}^{i=m} \sum_{j=1}^{j=m} x_{i j} d u_{i} d u_{j}+\sum_{i=1}^{m} x_{i} d^{2} u_{i}
\end{aligned}
$$

must lie in it. But as before, (8), (9), and $\sum_{i=1}^{m} x_{i} d u_{i}$ already lie in the tangent $S_{m}$ and therefore the only condition necessary is that the point

$$
\Sigma \Sigma x_{i j} d u_{i} d u_{j}
$$

should lie in it. This condition is

$$
\sum_{i, j=1}^{m} x_{i j} d u_{i} d u_{j}+\sum_{i=1}^{m} A_{i} x_{i}+A_{n+1} x=0 .
$$

It is evident, since there are $m-1$ independent quantities $d u_{1}: d u_{2}: d u_{3}: \cdots: d u_{m}$ at our disposal and $m+1$ quantities $A$, that equation (12) can always be satisfied if $2 m>n$. And if $2 m<n$, then the coordinates $x$ must be particular solutions of (12) if the osculating plane to a curve traced on $V_{m}$ lies in the tangent $S_{m}$. In this case, as in the preceding one, it is seen that if the point (11) lies in the tangent $S_{m}$, then the ratios $d^{2} u_{1}: d^{2} u_{2}: \cdots: d^{2} u_{m}$ can be so determined that (10) will lie in the line joining the points (8) and (9). Hence:

If through each point of a variety $V_{m}$ in $S_{n}$ there is a principal direction, then the coordinates must be particular solutions of the partial differential (12). The osculating planes of curves enveloped by these principal directions will lie in the tangent $S_{m}$ to the $V_{m}$.

From equation (12) we see that the principal directions passing through each point of a hypersurface $V_{n-1}$ in $S_{n}$ form a quadric cone of $n-2$ dimensions, and the principal 
directions through each point of a variety $V_{n-k}(2 k<n)$ form a cone of order $2^{k}$ and dimensions $n-2 k$. If $n=2 m-1$ and $k=m-1$ the number of principal directions will be equal to $2^{m-1}$. The curves enveloped by these directions correspond to the asymptotic curves on a surface in $S_{3}$ and in fact can be obtained by the consideration of conjugate directions just as in $S_{3}$. Take any two infinitely near points of $V_{m}$ and draw the tangent spaces $S_{m}$ to $V_{m}$ at each of these points. The two $S_{m}$ 's will intersect in a line and this line is said to be conjugate to the direction defined by the two points of tangency. Using the same method as in a previous paper* by the author, the condition that the two directions $d u$ and $\delta u$ be conjugate is found to be the vanishing of the $(m+2)$-rowed determinants of the matrix

$$
\left\|x, x_{1}, x_{2}, \cdots, x_{m}, \sum_{i} \sum_{j} x_{i j} \grave{d} u_{i} \delta u_{j}\right\| .
$$

The condition that the two conjugate directions should coincide is seen to be that the coordinates should satisfy equation (12). In this case then we have a new property of such curves, viz.: The tangent spaces $S_{m}$ to $V_{m}$ at two infinitely near points of the curve enveloped by the principal tangents intersect in the line joining the two points, that is, in a principal tangent. By an easy extension of the method used by Segret it can be shown that this is a property of such curves in general. Or it may be seen that if this property does hold in general there is an $S_{m+1}$ having two-point contact (i. e., tangent at two infinitely near points) with the variety $V_{m}$. Now the tangent at $x$ is determined by

$$
x, \quad x_{1}, \quad x_{2}, \quad x_{3}, \quad \cdots, \quad x_{m}
$$

and the tangent at the point is determined by

$$
\Sigma x_{i} d u_{i}, \quad \Sigma x_{1 j} d u_{j}, \quad \Sigma x_{2 j} d u_{j}, \quad \cdots, \quad \Sigma x_{m j} d u_{j} .
$$

It is seen at once that the tangent space (13) contains the point (14) and that the tangent space (15) contains the point

$$
\Sigma x_{i j} d u_{i} d u_{j}
$$

* Annals of Mathematics, vol. 13, p. 89.

+ Loc. cit. 
Now if the space (13) contains (11), the two tangent spaces will have the line defined by the direction $d u_{1}: d u_{2}: \cdots: d u_{m}$ in common. This is exactly condition (12). Hence:

The curves enveloped by the principal directions on a variety whose coordinates satisfy (12) are such that the tangent $S_{m}$ 's at two consecutive points intersect in the line joining the two points.

3. Let us now find the condition that a variety $V_{3}$ have through each point a tangent with four point contact with $V_{\mathbf{3}}$. The condition may be got by first finding the condition that curves exist on $V_{3}$ whose osculating $S_{3}$ at any point coincides with the tangent $S_{3}$ to $V_{3}$ at that point. This requires that the two points

$$
\begin{gathered}
\Sigma x_{i j} d u_{i} d u_{j}+\Sigma x_{i} d^{2} u_{i} \\
\Sigma x_{i j k} d u_{i} d u_{j} d u_{k}+3 \Sigma x_{i j} d u_{i} d^{2} u_{j}+\Sigma x_{i} d^{3} u_{i}
\end{gathered}
$$

should lie in the $S_{3}$ determined by the points

$$
x, \quad x_{1}, \quad x_{2}, x_{3} .
$$

This is equivalent to the relations

$$
A \Sigma x_{i j} d u_{i} d u_{j}+\sum_{1}^{3} B_{i} x_{i} d u_{i}+C x=0,
$$

(18) $A^{\prime}\left(\Sigma x_{i j k} d u_{i} d u_{j} d u_{k}+\Sigma x_{i j} d u_{i} d^{2} u_{j}\right)+\Sigma B_{i}{ }^{\prime} x_{i} d u_{i}+C^{\prime} x=0$.

In relation (12) the $d u_{i}$ define the direction of the curve. If (12) is satisfied, then the determinants of the matrix

$$
\left\|\Sigma x_{i j} d u_{i} d u_{j}, x, x_{1}, x_{2}, x_{3}\right\|
$$

must vanish. Now if we change $d u_{i}$ into $d u_{i}+\frac{1}{2} d^{2} u_{i,}$ this relation must still be satisfied; this leads to

$$
\left\|\Sigma x_{i j} d u_{i} d^{2} u_{j}, x, x_{1}, x_{2}, x_{3}\right\|=0,
$$

which used in (18) reduces that relation to

$$
A^{\prime} \Sigma x_{i j k} d u_{i} d u_{j} d u_{k}+\sum_{1}^{3} B_{i}{ }^{\prime} x_{i}+C^{\prime} x=0 .
$$

Hence:

If a variety $V_{3}$ is covered with a family of curves whose osculating $S_{3}$ at each point coincides with the tangent $S_{3}$ to $V_{3}$ at that point, then the coordinates must be solutions of two simultaneous partial differential equations, one of parabolic type of 
the second order and the other of the third order having coincident roots of its characteristic equation. The curves in question are the characteristics.

If equation (12) is satisfied, we saw that the tangents in the direction $d u_{i}$ have three-point contact with $V_{3}$. If in addition (19) is also satisfied, since (17) lies in the tangent $S_{3}$, the quantities $d^{3} u_{i}$ can be so chosen that (17) will lie on the line joining $x$ to $x+d x$. Hence:

When the coordinates of a spread $V_{3}$ satisfy the differential equations (12) and (19), through each point of the spread passes a tangent having four-point contact with the spread.

In the same manner it can be shown that the necessary and sufficient condition that a spread $V_{m}$ have in each point a tangent with $r$-point contact is that the coordinates should satisfy the $r-2$ equations

$$
\begin{aligned}
& A \Sigma \frac{\partial^{r-1} x}{\partial u_{i} \cdots \partial u_{r-1}} d u_{1} d u_{2} \cdots d u_{r-1}+\Sigma B_{i} x_{i}+C x=0, \\
& A^{(r-2) \Sigma} \frac{\partial^{2} x}{\partial u_{i} \partial u_{j}} d u_{i} d u_{j}+\Sigma B_{i}^{(r-2)} x_{i}+c^{r-2} x=0 .
\end{aligned}
$$

The osculating spaces to such curves always lie in the tangent $S_{m}$ to $V_{m}$. In particular when $r-1=m$ and the coordinates satisfy the system of equation (20), there is a curve passing through each point whose tangent lines have $(m+1)$-point contact with $V_{m}$. The osculating $S_{m}$ of the curve coincides with the tangent $S_{m}$ to $V_{m}$.

That equations (20) be satisfied requires $(n-m)(r-2)$ conditions. There are at our disposal $m-1$ ratios $d u_{1}$ : $d u_{2}: \cdots: d u_{m}$; therefore if the above number is less than or equal to $m-1$, the condition is always fulfilled. In case

$$
(n-m)(r-2)=m-1
$$

the number of curves through each point is finite. When $n=2 k-1, m=k, r=3$ the number of three point tangents passing through each point is $2^{k}$.

From (21) can be calculated the dimensions of $V_{m}$ such that it have a finite number of tangents through each point having $r$-point contact. In particular if the spread is to have a 4-point tangent through each point, $m=\frac{1}{3}(2 n+1)$.

Massachusetts Institute of Technology. 